\title{
Application of Stochastic Gradient Kernel in Watershed Segmentation to be used in Noisy Environment
}

\author{
Dibyendu Ghoshal \\ Associate Professor, Department of ECE \\ National Institute of Technology \\ Agartala, Tripura, Pin - 799055
}

\author{
Pinaki Pratim Acharjya \\ Assistant Professor, Department of CSE \\ Bengal Institute of Technology and Management \\ Santiniketan, West Bengal, Pin - 731236
}

\begin{abstract}
Morphological image processing has been widely used for segmentation of binary, grayscale and color images. To extend the concept of segmentation, an ordering of the data is required. In this research paper, an effective methodology for digital color image segmentation has been publicized with stochastic gradients and watershed algorithm. The results demonstrate that combining of these two strategies has been very helpful for image segmentation and for computer vision, even in noisy images. The efficiency of the proposed methodology has been explained by experimental results and statistical measurements.
\end{abstract}

\section{Keywords}

Image segmentation, image smoothing, stochastic gradient, watershed algorithm.

\section{INTRODUCTION}

Watershed based image segmentation [1-11] approach has found wide applications in various types of images which are not only confined in the optical spectrum but also in other frequency ranges like infrared, microwave and millimeter wave based imaging, viz radar imaging, satellite undergone through obtaining a gradient image because it helps to eliminate the major variations of the intensity of neighboring pixels. These gradient images are acquired by proposed by F. Mayer [5] by using standard high pass spatial filters $[15,18]$ like Sobel, LoG, Prewitt etc. These high pass linear spatial filters are capable of removing the noise in a medium scale but with the help of Canny edge detector these noise can be minimized to some appreciatable extent [16-17]. Stochastic gradient filters [15] have been found to be efficient reducing various types of noises (mostly adaptive noise) in a controlled manner. Hence the application of these stochastic gradient kernels in noisy images may be considered worthwhile and justified. All though a large number of research papers [1-13] are found in various journals, no study based on the stochastic gradient filter in watershed segmentation is available in published or online literature.

This paper is divided into a choice of sections. In section 2 stochastic gradient operators are publicized. Section 3 describes the gaussian filter for image smoothing. Section 4 introduces a brief description on watershed algorithm. Section 5 presents the proposed approach. The experimental results are discussed in section 6 and we imaging. The images are acquired in this practical field as mentioned above are usually subjected to further processing and analysis and these are prevarrent in radar imaging of various types. The images of the stellar objects [19-20] are acquired by satellites. The space trap already set off for long voyage, Hubble space telescope are most often found to be corrupted by different noises. A large number of digital images are transmitted through internet which includes both LAN and wireless channel. The images transmitted through internet are also prone to be effected by various types of additive and adaptive noises [15-16]. These noises are most often random in nature and they effects the major portion of any image and spatially they damage the border of the images and these damage sometime are found to be so devastating that it destroys the demarcating boundary line between the foreground image and there background.

Watershed algorithm has found a prominent place in image segmentation owing to its inherent property of having a stable output with minimum post processing. Hence it is obvious that all the types of problems arising out of quantification by the noise should be eliminated in the preliminary phases of watershed, i.e. mathematical morphological based image segmentation process. At the very concept of the watershed segmentation are normally

finish this paper with some concluding remarks with section 7.

\section{STOCHASTIC GRADIENT}

The gradient masks of standard high pass spatial filters perform poorly in noisy images. A better alternative is to design some masks, which take into the presence of noise in a controlled manner. The stochastic gradient operator can be obtained using definitions in (1).

$$
g_{1}(m, n) \triangleq u_{f}(m, n-1)-u_{b}(m, n+1)
$$

Stochastic gradient operators with different masks are shown in figure 1 to 6 . It is notable that for high signal noise ratio the filter waits decay rapidly.

\begin{tabular}{|c|c|c|}
\hline 0.776 & 0 & -0.776 \\
\hline 1.00 & 0 & -1.00 \\
\hline 0.776 & 0 & -0.776 \\
\hline
\end{tabular}

Fig 1: 3x3 Stochastic gradient mask, SNR=9. 


\begin{tabular}{|c|c|c|c|c|}
\hline 0.267 & 0.364 & 0 & -0.364 & -0.267 \\
\hline 0.373 & 0.562 & 0 & -0.562 & -0.373 \\
\hline 0.463 & 1.00 & 0 & -1.00 & -0.463 \\
\hline 0.373 & 0.562 & 0 & -0.562 & -0.373 \\
\hline 0.267 & 0.364 & 0 & -0.364 & -0.267 \\
\hline
\end{tabular}

Fig 2: $5 \times 5$ Stochastic gradient mask, SNR=9.

\begin{tabular}{|c|c|c|c|c|c|c|}
\hline 0.073 & 0.240 & 0.283 & 0 & -0.283 & -0.240 & -0.073 \\
\hline 0.104 & 0.213 & 0.348 & 0 & -0.348 & -0.213 & -0.104 \\
\hline 0.165 & 0.354 & 0.579 & 0 & -0.579 & -0.354 & -0.165 \\
\hline 0.195 & 0.463 & 1.00 & 0 & -1.00 & -0.463 & -0.195 \\
\hline 0.165 & 0.354 & 0.579 & 0 & -0.579 & -0.354 & -0.165 \\
\hline 0.104 & 0.213 & 0.348 & 0 & -0.348 & -0.213 & -0.104 \\
\hline 0.073 & 0.240 & 0.283 & 0 & -0.283 & -0.240 & -0.073 \\
\hline
\end{tabular}

Fig 3: 7x7 Stochastic gradient mask, SNR=9.

\begin{tabular}{|l|l|l|}
\hline 0.97 & 0 & -0.97 \\
\hline 1.00 & 0 & -1.00 \\
\hline 0.97 & 0 & -0.97 \\
\hline
\end{tabular}

Fig 4: 3x3 Stochastic gradient mask, SNR=1.

\begin{tabular}{|c|c|c|c|c|}
\hline 0.802 & 0.836 & 0 & -0.836 & -0.802 \\
\hline 0.845 & 0.897 & 0 & -0.897 & -0.845 \\
\hline 0.870 & 1.00 & 0 & -1.00 & -0.870 \\
\hline 0.845 & 0.897 & 0 & -0.897 & -0.845 \\
\hline 0.802 & 0.836 & 0 & -0.836 & -0.802 \\
\hline
\end{tabular}

Fig 5: 5x5 Stochastic gradient mask, SNR=1.

\begin{tabular}{|c|c|c|c|c|c|c|}
\hline 0.641 & 0.672 & 0.719 & 0 & -0.719 & -0.672 & -0.641 \\
\hline 0.656 & 0.719 & 0.781 & 0 & -0.781 & -0.719 & -0.656 \\
\hline 0.688 & 0.781 & 0.875 & 0 & -0.875 & -0.781 & -0.688 \\
\hline 0.703 & 0.831 & 1.00 & 0 & -1.00 & -0.831 & -0.703 \\
\hline 0.688 & 0.781 & 0.875 & 0 & -0.875 & -0.781 & -0.688 \\
\hline 0.656 & 0.719 & 0.781 & 0 & -0.781 & -0.719 & -0.656 \\
\hline 0.641 & 0.672 & 0.719 & 0 & -0.719 & -0.672 & -0.641 \\
\hline
\end{tabular}

Fig 6: 7x7 Stochastic gradient mask, SNR=1.

\section{GAUSSIAN FILTER FOR IMAGE SMOOTHING}

The Gaussian smoothing operator is a spatial low pass linear filter that is used to 'blur' images and remove detail and noise. Gaussian low pass filters have wide applicability in removing different types of additive and non additive noises. Out of these additive noises additive white gaussian noise (AWDL) Rayleigh noise, Erlang noise, exponential noise, uniform noise can be reduced by using Gaussian low pass filter. Besides impulse noises and speckle noises can be reduced to some small extent by using the same frequency domain low pass Gaussian filters. The degree of smoothing is determined by the standard deviation of the Gaussian. It is obvious that larger standard deviation Gaussians require larger convolution kernels. In below a Gaussian 7x7 mask which is used in proposed methodology for smoothing purpose of the gradient image is shown.

\begin{tabular}{|c|c|c|c|c|c|c|}
\hline 1 & 2 & 3 & 4 & 3 & 2 & 1 \\
\hline 2 & 3 & 4 & 7 & 4 & 3 & 2 \\
\hline 3 & 4 & 16 & 26 & 16 & 4 & 3 \\
\hline 4 & 7 & 26 & 41 & 26 & 7 & 4 \\
\hline 3 & 4 & 16 & 26 & 16 & 4 & 3 \\
\hline 2 & 3 & 4 & 7 & 4 & 3 & 2 \\
\hline 1 & 2 & 3 & 4 & 3 & 2 & 1 \\
\hline
\end{tabular}

Fig 7: Gaussian 7x7 mask.

\section{WATERSHED ALGORITHM}

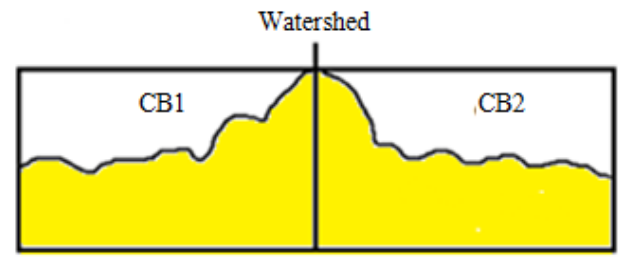

Fig 8: Watershed segmentation-local minima of gray level yield catchment basins, local maxima define the watershed lines.

Watershed algorithm is a tool for morphological image segmentation. A gray scale image can be interpreted as the topographic image of landscape. This is accomplished with the image intensity as an altitude. Using the features of these images, the technique of digital image processing called Watershed Transform. It consists in placing a water source in each regional minimum or catchment basins (CB), to flood the relief from sources, and build barriers when different sources are meeting. The resulting set of barriers constitutes a watershed by flooding, i.e., the set of pixels along which the gray levels changes sharply gives rise to a watershed edge. The mathematical formulation of watershed algorithm is sated below.

Assume, $M_{i}$ where $i=1$ to $\mathrm{n}$ be the set of coordinates points in the regional minima (catchment basins), of the image $P(x, y)$ and $C\left(M_{i}\right)$ be the coordinates points of catchment basins associated with the regional minima $M_{i}$

$$
T n=\{(s, t) \mid P(s, t)<n\}
$$

Where,

$T[n]=$ set of points in $P(x, y)$ which are lying below the plane $p(x, y)=n$

$\min , \max =$ minimum or maximum gray level value.

$n=$ stage of flooding varies from $\min +1$ to $\max +1$

Let $C_{n}\left(M_{1}\right)$ be the set of points in the catchment basin associated with $M_{l}$ that are flooded at stage $\mathrm{n}$.

$\operatorname{Cn}(M 1)=\cap\{C(M 1), T[n]\}$

Where,

$\operatorname{Cn}\left(M_{i}\right)= \begin{cases}1, & \text { if }(x, y) \in C\left(M_{i}\right) \text { and }(x, y) \in T[n] \\ 0, & \text { otherwise }\end{cases}$

(3) 
$C[n]$ is the union of flooded catchment basin portions at the stage $n$. Where,

$C[n]=C n(m 1) \cup C n(m 2) \ldots \ldots C n(m R)$

$C[\max +1]=C(m 1) \cup C(m 2) \ldots \ldots C(m R)$

If the algorithm keeps on increasing flooding level then $C_{n}\left(M_{i}\right)$ and $T[n]$ will either remain constant or increase. Algorithm initializes $C[\min +1]=T[\min +1]$, and then precedes recursively by assuming that at step n $C[n$ 1] has been constructed.

Let, $G$ is a set of connected components in $T[n]$ and for each connected component $g \in G[n]$, there possibilities will arise.

1. $g \cap C[n-1]$ is empty.

2. $g \cap C[n-1]$ contains one connected component of $C[n$ $1]$.

3. $g \cap C[n-1]$ contains more than one connected component of $C[n-1]$.

\section{PROPOSED METHODOLOGY}

Watershed segmentation is an effective method for image segmentation. To apply watershed segmentation to color images, first color images has been converted into gray scale images and with the help of spatial high pass filters gradient images has to generate next. The watershed algorithm is applied on that gradient image. In this process selection of a spatial high pass filters are needed as they decide the final segmented result. In this proposed methodology stochastic gradients of different masks and SNR have been used for generating gradient images as these gradient kernels are capable of removing the noise much better than the standard high pass filters. It has been also observed that direct apply of watershed algorithm on the gradient images results over segmentation. To reduce the scope of over segmentation problem smoothing technique is needed before applying watershed algorithm on the gradient images. A gaussian mask of $7 x 7$ is also introduced in this methodology for the smoothing purpose. And finally the watershed algorithm in applied to get the final segmented map or result image. The flow diagram of the proposed methodology is shown below in figure 9 .

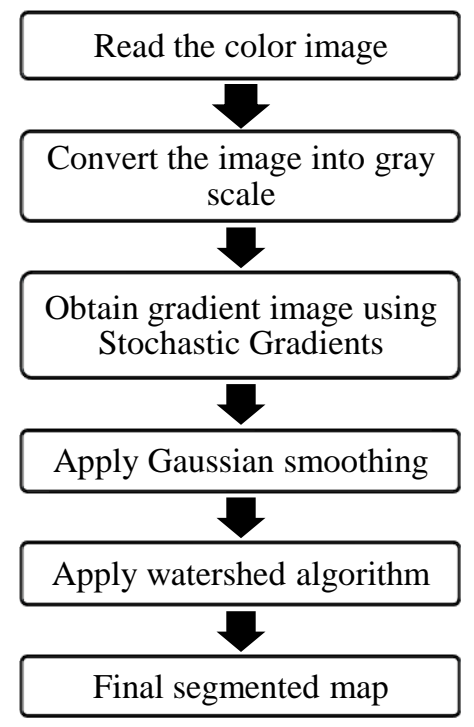

Fig 9: Flow diagram of the proposed methodology.

\section{EXPERIMENTAL RESULTS AND DISCUSSION}

Six different masks of stochastic gradients have been chosen to carry out the image segmentation process using the proposed method, $3 \times 3$ stochastic gradient masks with $\mathrm{SNR}=9$ and $\mathrm{SNR}=1,5 \times 5$ stochastic gradient masks with $\mathrm{SNR}=9$ and $\mathrm{SNR}=1$ and $7 \times 7$ stochastic gradient mask with $\mathrm{SNR}=9$ and $\mathrm{SNR}=1$. SNR (Signal to noise ratio) is a measure used in science and engineering that compares the level of a desired signal to the level of background noise. It is defined as the ratio of signal power to the noise power. The proposed approach has been applied on two real life images of "Lena" and "Laure" of 512 x 512 and 256 x 256 dimensions respectively. The original image is shown in figure 10 and 17. The final segmented images have been shown below from Figure 11 to Figure 16 and from figure 18 to 23 respectively for "Lena" and "Laure". The watershed ridge lines have been imposed on the final segmented images. The statistical analyses of the segmented images have been calculated in respective of Entropy, PSNR and MSE and are shown in table 1. It is observed from the segmented images with $\mathrm{SNR}=1$ are less over segmented then the images acquired with the masks having SNR 9. From the statistical point of view, the segmented image with $7 \times 7$ stochastic gradient mask with MSE $=1$, produce lesser Entropy and PSNR with higher MSE. The segmented image with $3 \times 3$ stochastic gradient mask with $\mathrm{MSE}=1$, produce higher Entropy and PSNR with lesser MSE. It is also observed that $7 \times 7$ stochastic gradient mask with $\mathrm{SNR}=1$ produce better results in respective of the statistical analysis and human perception. Thus, in this research work, $7 \times 7$ stochastic gradient mask has been found to be most efficient in reducing various types of noises. Experimental results presented in this paper are obtained by using MATLAB. 


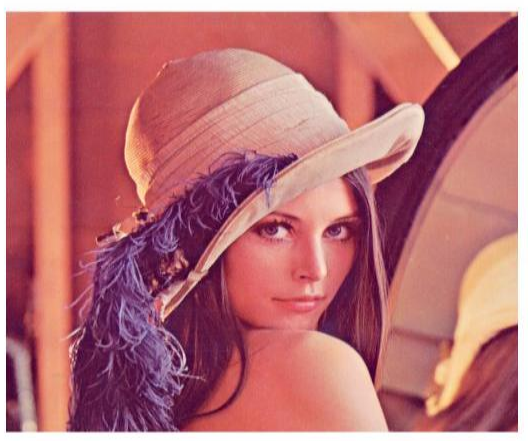

Fig 10: Original Image of Lena.

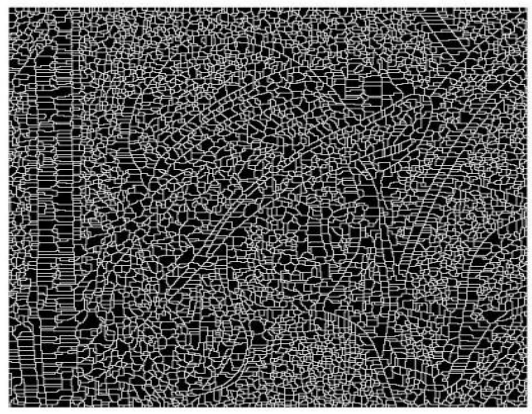

Fig 11: Segmented image of Lena with 3x3 Stochastic gradient mask, $\mathrm{SNR}=9$.

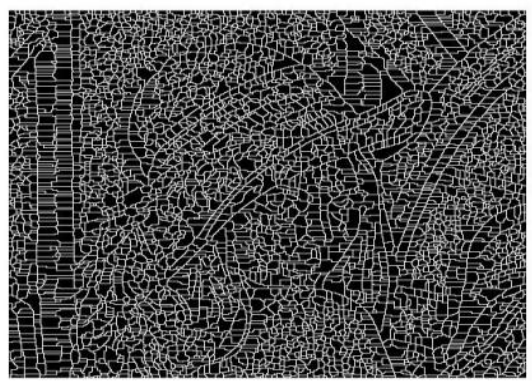

Fig 12: Segmented image of Lena with $5 \times 5$ Stochastic gradient mask, $\mathrm{SNR}=9$.

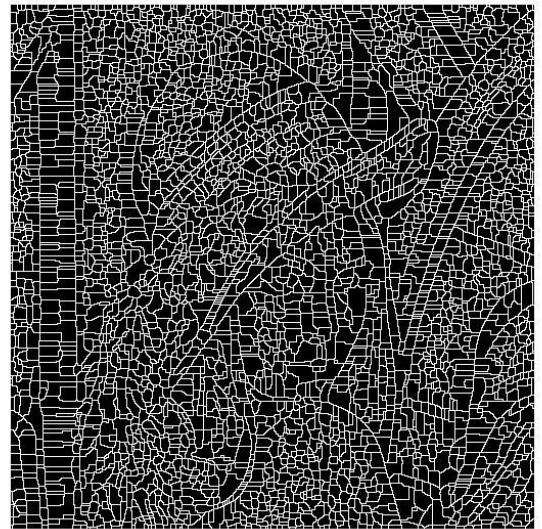

Fig 13: Segmented image of Lena with $7 \times 7$ Stochastic gradient mask, SNR=9.

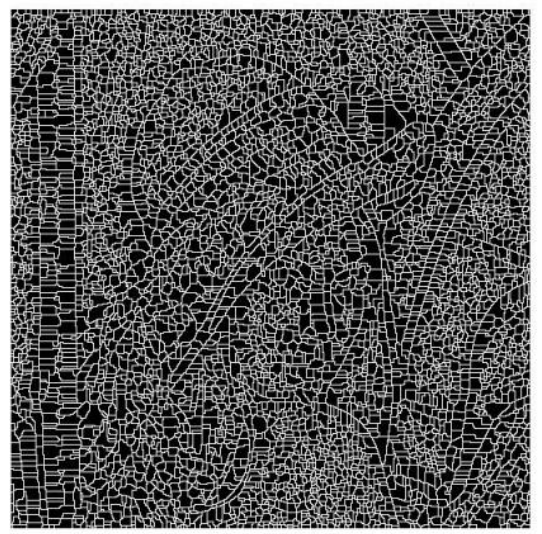

Fig 14: Segmented image of Lena with $3 \times 3$ Stochastic gradient mask, $\mathrm{SNR}=1$. 


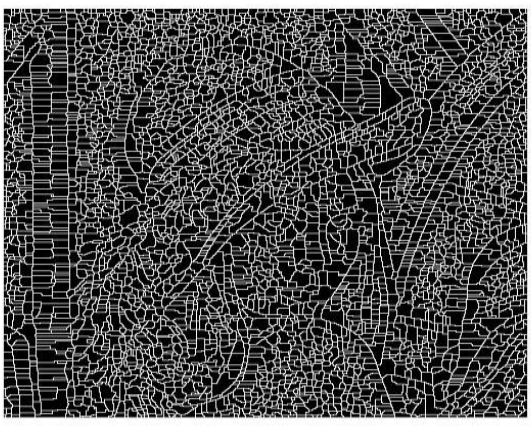

Fig 15: Segmented image of Lena with $5 \times 5$ Stochastic gradient mask, $\mathrm{SNR}=1$.

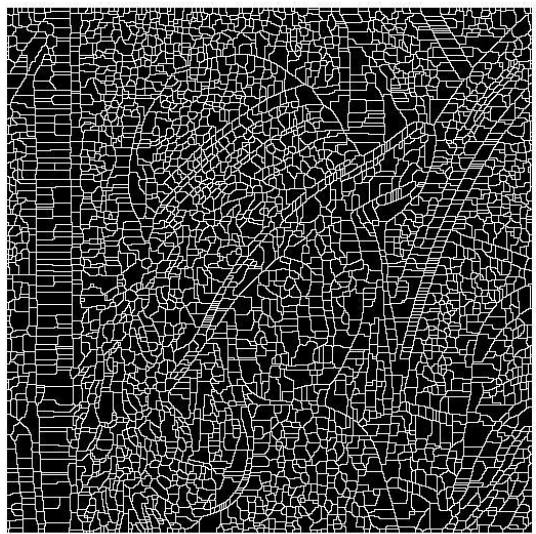

Fig 16: Segmented image of Lena with $7 \times 7$ Stochastic gradient mask, $\mathrm{SNR}=1$.

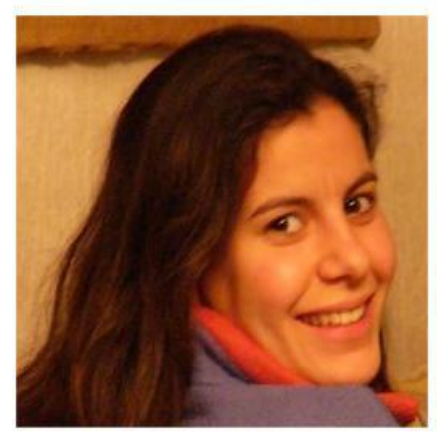

Fig 17: Original Image of Laure.

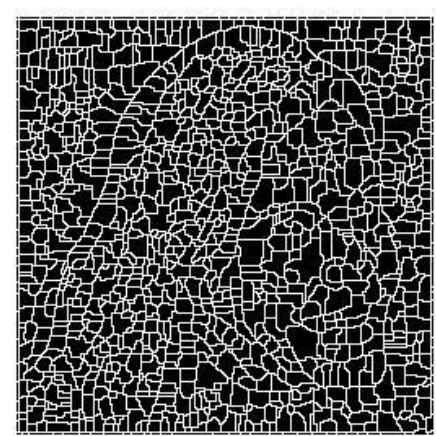

Fig 18: Segmented image of Laure with $3 \times 3$ Stochastic gradient mask, $\mathrm{SNR}=9$.

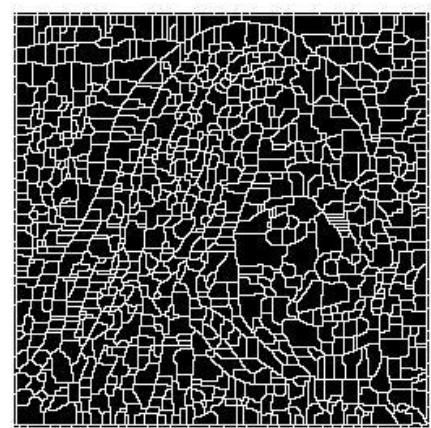

Fig 19: Segmented image of Laure with $5 \times 5$ Stochastic gradient mask, $\mathrm{SNR}=9$.

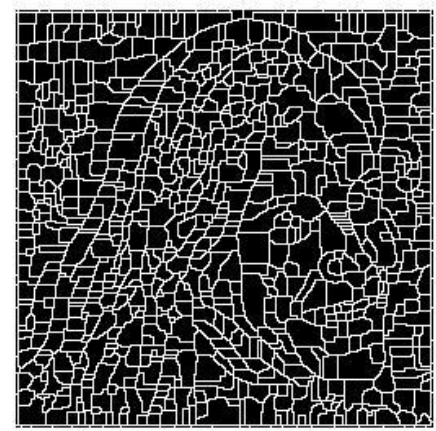

Fig 20: Segmented image of Laure with $7 \times 7$ Stochastic gradient mask, $\mathrm{SNR}=9$. 


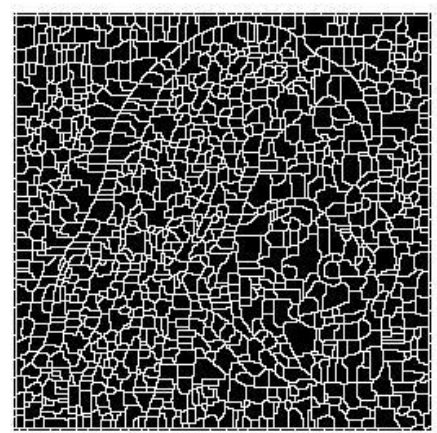

Fig 21: Segmented image of Laure with $3 \times 3$ Stochastic gradient mask, $\mathrm{SNR}=1$.

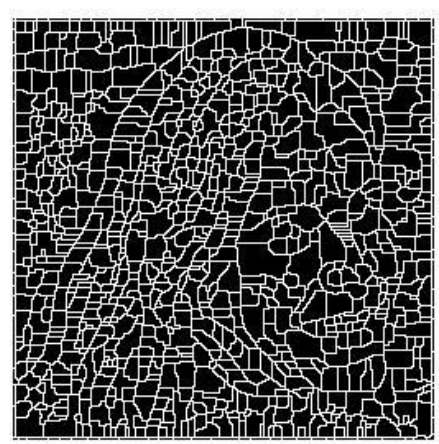

Fig 22: Segmented image of Laure with $5 \times 5$ Stochastic gradient mask, $\mathrm{SNR}=1$.

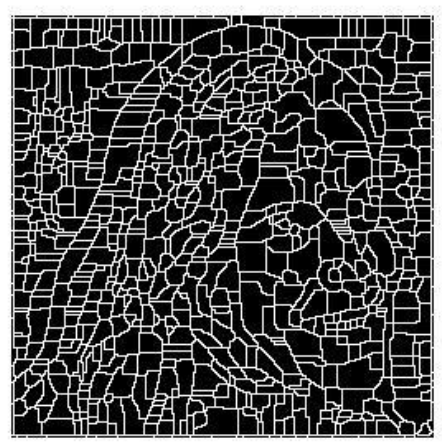

Fig 23: Segmented image of Laure with $7 \times 7$ Stochastic gradient mask, $\mathrm{SNR}=1$.
Table I- statistical measurement

\begin{tabular}{|c|c|c|c|}
\hline Image & Entropy & PSNR & MSE \\
\hline Lena & 3.1801 & 7.6592 & $1.1147 \mathrm{e}+004$ \\
$(3 \times 3$ mask, \\
SNR=9) & & & \\
\hline Lena & 3.1621 & 7.6383 & $1.1201 \mathrm{e}+004$ \\
$(5 \times 5$ mask, \\
SNR=9) & & & \\
\hline Lena & 3.1414 & 7.6251 & $1.1235 \mathrm{e}+004$ \\
$(7 \times 7$ mask, & & & \\
SNR=9) & & & \\
\hline Lena & 3.1802 & 7.6622 & $1.1139 \mathrm{e}+004$ \\
$(3 \times 3$ mask, \\
SNR=1)
\end{tabular}




\section{CONCLUSION}

Image segmentation is an essential process for most sub sequent image analysis tasks. In this research paper, an effective methodology for digital color image segmentation has been publicized with stochastic gradients and watershed algorithm. The proposed methodology has been successfully tested on a digital color image. The results demonstrate that combining stochastic gradients with watershed algorithm can be very helpful for image segmentation and for computer vision, even in noisy images. The experimental results and statistical measurements confirm the efficiency of the proposed methodology.

\section{DEDICATION}

One of the others (Dibyendu Ghoshal) dedicates the entire study to the loveliest and loving memory of his only one and younger sister Kumari Sumita Ghoshal who herself was a gem of the scholars, a symbol of wisdom and art, peerless beauty and simplicity, unfathomable knowledge and generosity.

\section{REFERENCES}

[1] L. Vincent, "Morphological grayscale reconstruction in image analysis: Applications and efficient algorithms," IEEE Transactions on Image Processing, vol. 2, pp. 176-201, 1993.

[2] L. Vincent and P. Soille, "Watersheds in digital spaces: an efficient algorithm based on immersion simulations," IEEE transactions on pattern analysis and machine intelligence, vol. 13, pp. 583-598, 1991.

[3] S. Beucher and C. Lantuejoul, "Use of watersheds in contour detection," 1979.

[4] F. Meyer and S. Beucher, "Morphological segmentation," Journal of visual communication and image representation, vol. 1, pp. 21-46, 1990.

[5] F. Meyer, "Topographic distance and watershed lines," Signal Processing, vol. 38, pp. 113-125, 1994.

[6] L. Vincent, Algorithmes morphologiques a base de files d'attente et de lacets: extension aux graphes: Paris, 1990.

[7] A. N. Moga and M. Gabbouj, "Parallel image component labeling with watershed transformation," IEEE transactions on pattern analysis and machine intelligence, vol. 19, pp. 441-450, 1997.

[8] J. Roerdink and A. Meijster, "The watershed transform: Definitions, algorithms and parallelization strategies," Mathematical Morphology, vol. 41, pp. 187-S28, 2000.
[9] P. Soille, Morphological image analysis: principles and applications: Springer-Verlag New York, Inc. Secaucus, NJ, USA, 1999.

[10] J. Serra, Image analysis and mathematical morphology: Academic Press, Inc. Orlando, FL, USA, 1983.

[11] J. Serra and L. Vincent, "An overview of morphological filtering," Circuits, Systems, and Signal Processing, vol. 11, pp. 47-108, 1992.

[12] W. J. Niessen, K. L. Vincken, J. A. Weickert, and M. A. Viergever, "Nonlinear multiscale representations for image segmentation," Computer Vision and Image Understanding, vol. 66, pp. 233-245, 1997.

[13] M. Berouti, R. Schwartz, and J. Makhoul, "Enhancement of Speech Corrupted by Acoustic Noise," in IEEE International Conference on Acoustics, Speech, and Signal Processing, pp. 208211, 1979. H. Digabel and C. Lantuejoul, "Iterative algorithms," Quantitative Analysis of Microstructures in Materials Sciences, Biology and Medicine, pp. 8599, 1977.

[14] S. F. Boll, "Suppression of Acoustic Noise in Speech Using Spectral Subtraction," IEEE Transactions on Acoustics, Speech and Signal Processing, vol. 27, no. 2, pp. 113-120, 1979.

[15] A.K.Jain, "Fundamentals of digital image processing", Second Edition, Prentice Hall, 2002.

[16] Canny, J., A Computational Approach To Edge Detection, IEEE Trans. Pattern Analysis and Machine Intelligence, 8(6):679-698, 1986.

[17] R. Deriche, Using Canny's criteria to derive a recursively implemented optimal edge detector, Int. J. Computer Vision, Vol. 1, pp. 167-187, April 1987.

[18] C. Gonzalez, Richard E. Woods, "Digital Image Processing", 2nd Edition, Addison Wesley Pub. Co, 2002.

[19] Greene, Thomas P.; Wilking, Bruce A.; Andre, Philippe; Young, Erick T.; Lada, Charles J , "Further mid-infrared study of the rho Ophiuchi cloud young stellar population: Luminosities and masses of premain-sequence stars", The Astrophysical Journal, vol. 434, pp. 614-626, 1994.

[20] Andre, Philippe; Ward-Thompson, Derek; Barsony, Mary, "Submillimeter continuum observations of Rho Ophiuchi A - The candidate protostar VLA 1623 and prestellar clumps", The Astrophysical Journal, vol. 406, pp. 122-141, 1993. 